\title{
Observing Catalyst Structures and Dynamics at Atomic-Resolution
}

M. Ek ${ }^{1}$, L. P. Hansen ${ }^{1}$, F.-R. Chen ${ }^{2}$, D. van Dyck ${ }^{3}$, C. Kisielowski ${ }^{4}$, J. R. Jinschek ${ }^{5}$, S. Helveg $^{1}$.

1. Haldor Topsoe A/S, Lyngby, Denmark.

2. Department of Engineering and System Science, National Tsing-Hua University, Hsin Chu, Taiwan.

3. Departments of Physics, EMAT, University of Antwerp, Antwerp, Belgium.

4. Molecular Foundry, Lawrence Berkeley National Laboratory, Berkeley, USA.

5. Department of Materials Science and Engineering \& Center for Electron Microscopy and Analysis (CEMAS), Ohio State University, USA.

Over the past decade, electron microscopy has become indispensable for studying heterogeneous catalysts at the atomic-scale [1]. The ability to acquire atomic-resolution images with single-atom sensitivity has opened up for unprecedented insight into catalyst structures and dynamics. However, the progress has also shown that observations at the atomic-level require an intense electron illumination that generally alters the catalyst during observation. The electron-induced alterations are particular pronounced at the catalyst surface as they expose a variety of sites of reduced atomic coordination. In the quest to suppress electron-induced alterations and to enable chemical meaningful observations, it therefore becomes mandatory to exercise control over the electron dose, dose-rate and energy.

Here, we demonstrate low dose-rate in-line electron holography as a viable concept for atomicresolution observations of catalysts in the genuine state [2-5]. The imaging scheme employs bright field transmission electron microscopy (TEM) as the most efficient way to detect single atoms using the fewest elastically scattered electrons. The image acquisition is done with low electron dose-rates of down to $1-100 \mathrm{e}^{-} \AA^{-2} \mathrm{~s}^{-1}$ to induce only weak object excitations and to offer time for reversible object restoration between successively delivered electrons. As a result, the individual atomic-resolution images are dominated by noise, and recovery of image signal can be accomplished by averaging over a series of consecutively acquired images of the object. For this purpose, in-line holography based on focal image series is particular attractive as residual aberrations are corrected and the exit wave (EW) function is recovered with enhanced signal. The significance of low dose-rate in-line electron holography is illustrated by observations of a $\mathrm{Co}_{3} \mathrm{O}_{4}$ catalyst material. Specifically, Figure 1 shows that lower dose-rates sharpen up the atomic column contrast and reduce smearing of the surface contrast. This suppression of beam-induced atom dynamics facilitates a quantitative interpretation of the EW.

Applications of low dose-rate in-line holography to heterogeneous catalysts will be outlined. One example focusses on carbon-supported single-layer $\mathrm{MoS}_{2}$ nanocrystals with edge-attached Co promoter atoms [6], which is relevant for hydrodesulfurization processes in oil refineries. A quantitative analysis of EWs allows the stoichiometric arrangement in the Co-Mo-S nanocrystals to be retrieved in three dimensions at single-atom sensitivity, as in [5]. The low dose-rate concept also benefits in situ electron microscopy. Such observations will be outlined for $\mathrm{VO}_{\mathrm{x}}$ supported on anatase- $\mathrm{TiO}_{2}$ nanoparticles $\left(\mathrm{VO}_{\mathrm{x}} / \mathrm{TiO}_{2}\right)$, which is widely applied for catalytically reducing $\mathrm{NO}_{\mathrm{x}}$ emissions that are harmful to human health and ecosystems [7]. Specifically, image series were recorded using low dose-rates for which the $\mathrm{V} \mathrm{L}_{3}$ ionization edge remained constant, indicating that electron-induced oxide alterations were suppressed. Surprisingly, the images reveal a facet-dependent and reversible order-disorder transformation of the outermost atomic layers of the $\mathrm{VO}_{\mathrm{x}} / \mathrm{TiO}_{2}$ catalyst under alternating oxidizing and reducing environments. 
References:

[1] S. Helveg, J. Catal. 328 (2015) p. 102.

[2] C Kisielowski et al, Phys Rev B 88 (2013) p. 024305.

[3] S. Helveg et al, Micron 68 (2014), p. 176.

[4] C. Kisielowski et al, Adv. Struct. Chem. Imag. 2 (2016), p. 13.

[5] F.-R. Chen, D. van Dyck, C. Kisielowski, Nature Comm. 7 (2016) p. 10603.

[6] F.-R. Chen et al (2018) in prep.

[7] M. Ek et al, Nature Comm. 8 (2017) p. 305.

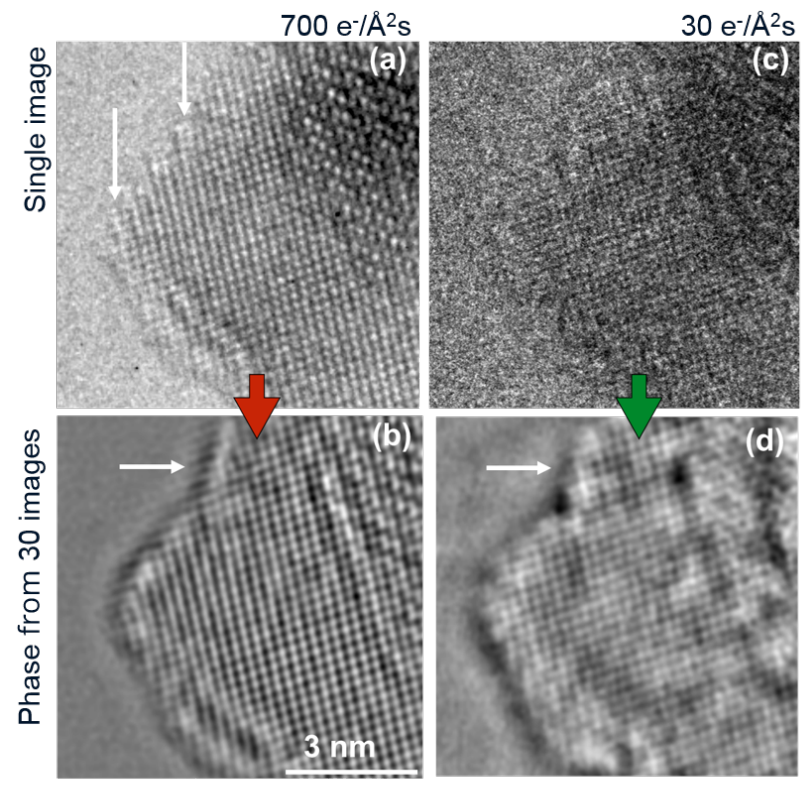

Figure 1. Low dose-rate in-line holography of a $\mathrm{Co}_{3} \mathrm{O}_{4}$ catalyst material. (a, c) Single HRTEM images of $\mathrm{Co}_{3} \mathrm{O}_{4}$. (b,d) Exit wave phase images of $\mathrm{Co}_{3} \mathrm{O}_{4}$. All images obtained in $2.1 \mathrm{mbar} \mathrm{CO}$. The dose-rates are vastly different $\left(700 \mathrm{e}^{-} \AA^{-2} \mathrm{~s}^{-1}\right.$ in $\mathrm{a}, \mathrm{b}$ and $30 \mathrm{e}^{-} \AA^{-2} \mathrm{~s}^{-1}$ in $\left.\mathrm{c}, \mathrm{d}\right)$. Arrows emphasize a streaky contrast at the high dose-rate in (b) which is absent at the low dose-rate in (d). This streaky contrast is therefore attributed to beam-induced atom displacements. From [3]. 